\title{
Scenari e ruoli della Medicina di Laboratorio al tempo della crisi: da Modena a Salerno e oltre
}

\author{
Laboratory Medicine: scenarios and roles of Laboratory Medicine in the time of crisis
}

\section{Piero Cappelletti}

Ricevuto: 12 novembre 2012 / Accettato: 19 novembre 2012

(C) Springer-Verlag Italia 2012

Riassunto Questo numero della Rivista Italiana della Medicina di Laboratorio contiene i contributi derivanti dalle relazioni della Sessione Plenaria inaugurale del $26^{\circ}$ Congresso Nazionale della SIMeL (Salerno 3-5 ottobre 2012), dal significativo titolo "Medicina di Laboratorio: scenari e ruoli al tempo della crisi". Il congresso ha esaminato lo stato della medicina di laboratorio italiana, stretta tra le difficoltà finanziarie, i profondi cambiamenti organizzativi, il continuo progresso scientifico e diagnostico e il mutamento professionale, sociale e culturale che da tutto ciò deriva. In questo quadro, quattro ruoli principali sono stati descritti: il ruolo di sacerdote della tecnologia comprende innovazione tecnologica, selezione di tecnologie e partner, controllo di qualità, valutazione delle forme di gestione; il ruolo organizzativo-gestionale consiste nella pianificazione strategica, organizzazione e gestione del laboratorio e dei suoi addetti, con particolare attenzione ai processi, al prodotto e al loro controllo; il ruolo di consulente clinico si esplica come consulenza nella richiesta e nell'interpretazione dei casi singoli, ma

P. Cappelletti (凶)

IRCCS CRO Aviano

Via Franco Gallini 2, 33081 Aviano (PN), Italy

E-mail: piero.cappelletti@cro.it anche di gruppi interdisciplinari, in particolare a fini di audit clinico; il ruolo di attore del governo clinico si traduce nella definizione collaborativa di "evidenze", linee guida, EBLM e PDTA, oltre che nella formazione di specialisti e MMG. L'insieme delle relazioni mostra la declinazione attuale della Medicina di Laboratorio e le caratteristiche dei professionisti che si riconoscono nella SIMeL: apertura all'innovazione e capacità di giudicarla, lavoro all'interno del laboratorio e all'interfaccia del processo di produzione dell'informazione diagnostica, coscienza delle caratteristiche competenziali e innovative dell'equipe del laboratorio, capacità relazionali e collaborative, attori principali della costruzione di "evidenze" scientifiche e gestionali, consapevolezza che i tempi richiedono soluzioni radicali e non piccolo cabotaggio e, soprattutto, il coraggio di veleggiare nei mari dell'incertezza ma anche delle opportunità.

Parole chiave Medicina di Laboratorio - innovazione • tecnologia $\cdot$ organizzazione $\cdot$ consulenza clinica $\cdot$ governo clinico

Summary This number of the Italian Journal of Laboratory Medicine presents four reviews, derived from the reports of the Plenary Session of the 26st National Congress of the Italian Society of Laboratory Medicine (SIMeL), held in Salerno October 3-5, 2012. The Congress "Laboratory Medicine: scenarios and roles in the crisis time" examined the state of art of laboratory 
medicine in Italy, affected by financial cuts, hard organizational changes, continuous scientific and diagnostic progress, and the consequent professional, social and cultural transformation. In this scenario, four principal roles for laboratory professionals were identified: the "priests of technology" related to the technologic innovation and its selection, evaluation and control; the "manager" as strategizing, organizing, managing workforce, processes and perspectives of the Laboratory; the "consultant" as partner in selection and interpretation of clinical testing in the individual patient and as essential part in the clinical audits; and the "actor of clinical governance" engaged in the definition of "evidences", evidence-based laboratory medicine, guidelines and care pathways. Taken together, the reviews show what the SIMeL professional think about Laboratory Medicine and its future in the crisis times: openness to innovation and knowledge for its evaluation, awareness of the necessary disruptive changing and of the unchangeable essentials of discipline, willingness to work at the clinic-laboratory interface, ability to establish evidences and relationships for a shared governance of the new healthcare of the $21^{\text {st }}$ century, and, overall, the courage for navigating uncertain seas of challenges and opportunities.

Key words Laboratory Medicine - innovation · technology $\cdot$ management $\cdot$ clinical consultant $\cdot$ clinical governance

\section{Introduzione}

Questo numero della Rivista Italiana della Medicina di Laboratorio contiene i contributi derivanti dalle relazioni della Sessione Plenaria inaugurale del $26^{\circ}$ Congresso Nazionale della Società Italiana Medicina di Laboratorio (SIMeL) [Salerno 3-5 ottobre 2012], dal significativo titolo "Medicina di Laboratorio: scenari e ruoli al tempo della crisi". Il Congresso ha esaminato lo stato della medicina di laboratorio italiana, stretta tra le difficoltà finanziarie, i profondi cambiamenti organizzativi, il continuo progresso scientifico e diagnostico e il mutamento professionale, sociale e culturale che da tutto ciò deriva. Le aree disciplinari investigate hanno spaziato dall'ematologia, autoimmunologia, medicina dello sport, medicina molecolare, allergologia e gastroenterologia all'endocrinologia, neurologia, cardiologia, oncologia, malattie infettive e nefrologia. Tuttavia, la sessione plenaria ha voluto indagare in termini generali scenari e ruoli della medicina di laboratorio in Italia oggi.

Si è trattato di una valutazione delle condizioni di vita della disciplina, a distanza di circa un decennio da quella operata nel 2004 al Congresso di Modena, che resta a tutt'oggi l'evento fondamentale e insuperato di presa di coscienza della professione dei cambiamenti epocali intervenuti al passaggio del millennio e i cui effetti perdureranno per diversi decenni. Nel "Patto per la modernizzazione e l'umanizzazione della Medicina di Laboratorio" [1], la Società, a nome della parte più avvertita della Medicina di Laboratorio, prendeva atto di una necessaria razionalizzazione della medicina di laboratorio in Italia alla luce del numero e della distribuzione dei laboratori e dei professionisti e dei travolgimenti in corso nel mondo occidentale (Canada, USA, UK), ma rivendicava il ruolo di attore primario nella costruzione di un'organizzazione più rispondente ai criteri di efficienza, economicità e produttività e, contemporaneamente, nella garanzia della qualità dell'intero processo di produzione di risultati, ovunque prodotti (neutralità del sito e governo di tutte le modalità in cui si declina la risposta al quesito clinico). Nel "Patto" i professionisti si impegnavano, sotto l'aspetto analitico, alla qualità/sicurezza del risultato e, sotto quello pre-analitico, alla qualità del campione e del suo trattamento, ma anche del paziente, comprendendo l'ambulatorietà come campo di competenza professionale e, in generale, delle condizioni per garantire l'appropriatezza (contribuendo a definire "evidenze" e linee guida); sotto l'aspetto post-analitico, a interpretazioni, suggerimenti e consulenza, nello specifico paziente e, in termini generali, attraverso il lavoro di gruppo con la misura degli outcomes e gli audit. Inoltre, si impegnavano a offrire ai cittadini e ai clinici il meglio della moderna tecnologia, valutandola in termini interattivi per le ricadute cliniche e sociali, e consci dell'importanza della diagnostica nel processo assistenziale a rispondere compiutamente ai bisogni "umani" espliciti e impliciti nella richiesta di esame di laboratorio. D'altra parte chiedevano che, come la medicina e la medicina di laboratorio si fondano oggi sulle "evidenze", così anche le razionalizzazioni e modernizzazioni fossero fondate sulle "evidenze".

\section{Scenari e Ruoli}

Il quadro generale che contiene e, almeno in parte, determina la trasformazione della Medicina di Laboratorio nel ventunesimo secolo è stato precedentemente esaminato, sotto il profilo culturale per l'irrompere del "soggetto" postmoderno nell'arena filosofica e sociale [2], che determina la nascita dell'esigente al posto del paziente; sotto il profilo organizzativo e finanziario delle organizzazioni sanitarie, centrate sul paziente-cittadino, ma attanagliate dai costi esponenzialmente e insostenibilmente crescenti [3]; sotto il profilo dell'organizzazione scientifica, professionale e sociale della Medicina di Laboratorio, in cui 
opportunità e paure si mescolano indissolubilmente nelle prospettive future della disciplina [4].

Secondo la lezione di Clayton Christensen [5], l'information and communication technology e la miniaturizzazione dei componenti che consentono POCT (point-ofcare testing) e LOC (lab-on-chip), a breve impiantabili (personal profile chip, PPC) e in grado di analisi geniche nella singola cellula (phenotypic monitoring gene chip, PMGC), hanno determinato l'innovazione radicale (disruptive innovation) in Medicina di Laboratorio, con l'applicazione della generale rottura dell'unità spazio-temporale del rapporto medico-paziente al campo specifico della disciplina, rottura che si sostanzia nella contemporanea pressione di centralizzazioni e decentralizzazioni. Questo travolgimento pone i grandi temi di continuare a garantire affidabilità e qualità/sicurezza dei risultati in ogni sito di produzione, di operare all'interfaccia clinicalaboratorio a fronte della sua geometrica amplificazione e di "traslare" correttamente nuovi metodi e marcatori dalla ricerca alla cura comprendendone e, per quanto possibile, guidandone le potenzialità "trasformative" (Fig. 1).

La ragion d'essere della Medicina di Laboratorio, in questo scenario, rimane quella di fornire informazioni e consulenza, per la salute degli esseri umani, attraverso una variegata e complessa rete di mezzi tecnici e disciplinari, su materiali di provenienza umana (ECLM 1993 ISO 15189:2003E). E la qualità dell'informazione diagnostica continua a essere definita dagli attributi "valida", "sicura", "appropriata" e "conveniente", con un'attenzione probabilmente maggiore al contenuto organizzativo e sociale, centrato sul paziente, di quest'ultima specificazione [6]. L'elaborazione teorica della SIMeL, attorno alle caratteristiche della produzione di un'informazione diagnostica di siffatta qualità, contiene le riflessioni di Lundberg sul "brain-to-brain loop" e di Büttner sul concetto di interfaccia clinica-laboratorio, ma le arricchisce di una biunivoca comunicazione tra cervelli, da un lato quello del "clinico" sempre più spesso sostituito da una pluralità di attori dei percorsi diagnostici e dall'altro quello della rete di cervelli dell'equipe che opera in laboratorio (Fig. 2). Questo aspetto di riflessione teorica, frutto dell'apporto di molti a cominciare da Giancarlo Fiorucci, è del tutto originale nel panorama della discussione nazionale e internazionale intorno al cosiddetto total testing process, anche quando ripensato per ricomprenderne il rapporto con il paziente [7]. L'informazione diagnostica, in essenza, è frutto dell'attività coordinata e collaborativa di un'equipe complessa e articolata nelle sue responsabilità e autonomie, tenuta insieme dalla sua cultura e mission e dalla responsabilità clinico-organizzativa e leadership. Le trasformazioni "sociali" di questa organizzazione in termini "macro", per il mutamento radicale del contesto in cui opera, e in termini "micro", per il cambiamento dei ruoli delle professioni che la compongono, hanno determinato importanti modifiche nelle competenze richieste a tutti i componenti dell'equipe. In particolare, si è rotta la netta separazione tra attività analitica e lavoro all'interfaccia, richiedendo a tutti i ruoli, oltre che le competenze specifiche, quelle comunicative [6].

In questo contesto nascono nuovi ruoli e altri devono essere "inventati": clinical science manager; quality manager, risk manager, education \& training officer and practice tutor, LIS manager, POCT manager...; advanced practitioner scientist, clinical diagnostic scientist, skills mix manager, audit manager, ICT manager...; translational scientist, comunication scientist, cultural transformer [4]. Per "ruolo" s'intende un insieme di comportamenti richiesti, in termini di attività, e attesi, in termini di integrazione alla cultura dell'organizzazione. I comportamenti correlati alle attività riferiscono ad azioni specifiche, indipendentemente dalle responsabilità. La

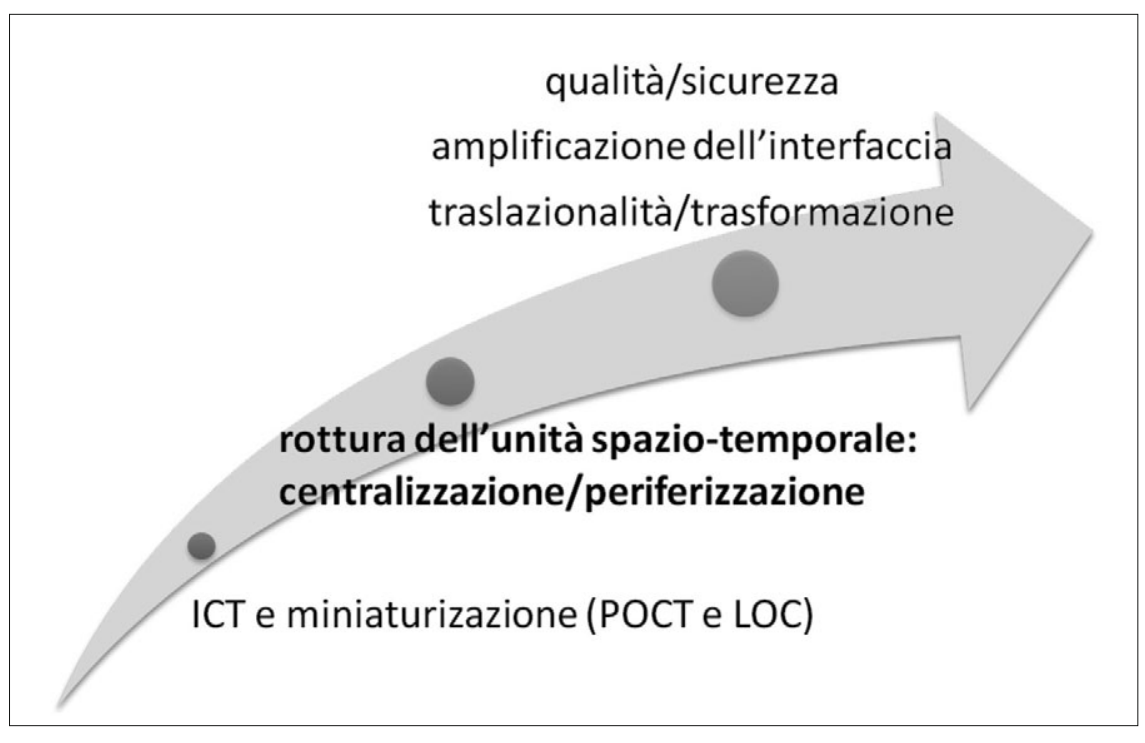

Fig. 1 L'information and comunication technology (ICT) e la miniaturizzazione che rendono possibili POCT (point-of-care testing) e LOC (lab-on-chip) determinano la rottura dell'unità spazio-temporale del rapporto medicopaziente, che si declina in Medicina di Laboratorio nelle contemporanee e contrapposte tensioni verso le centralizzazioni e le decentralizzazioni. Ciò pone i grandi temi della qualità/sicurezza in tutti i siti di produzione di risultati, dell'operatività all'interfaccia clinica-laboratorio enormemente amplificata e del governo della "traslazione" della ricerca in cura e del suo potenziale "trasformativo" di organizzazioni ed esiti clinici 


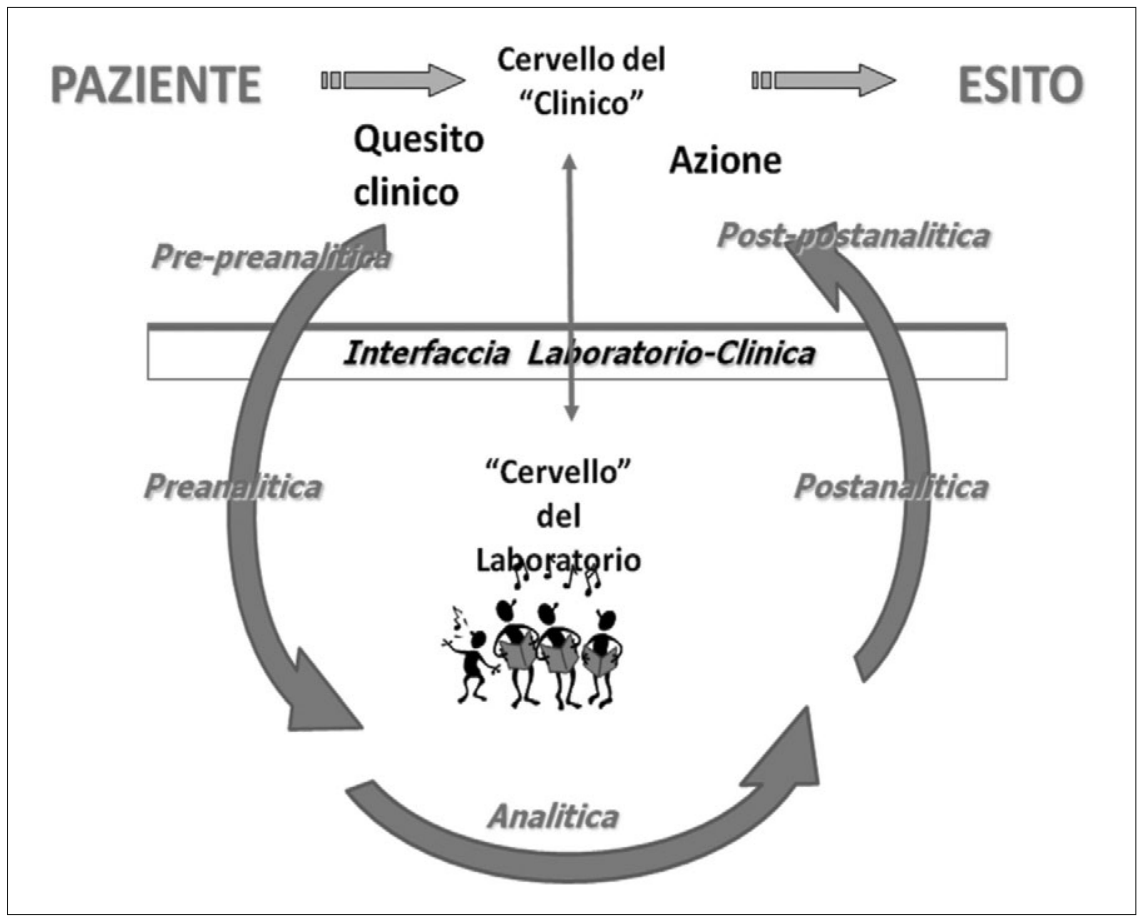

Fig. 2. L'informazione diagnostica è frutto dell'attività coordinata e collaborativa di un'equipe complessa e articolata nelle sue responsabilità e autonomie, tenuta insieme dalla sua cultura e mission e dalla responsabilità clinicoorganizzativa e dalla leadership

differenza tra ruolo e mansione sta nel fatto che il primo stabilisce le attività, la seconda i compiti e le responsabilità. La specificità della competenza riferisce alla cultura dell'organizzazione, non alla mansione. In questo senso, e nel quadro di mutamento dianzi delineato, a confini definitivi e permanenti del "ruolo" se ne sostituiscono di mobili, da definire e stabilizzare. I contenuti sono intesi come uno spazio "discrezionale" che determina rischio e incertezza e richiede comunicazione e negoziazione. Per ridefinire e inventare ruoli è necessario: migliorare le competenze; stabilizzare gli aspetti oggettivi del ruolo; capire e chiarire il mandato; definire i legami organizzativi; stabilizzare lo status; valutarne gli aspetti soggettivi: obiettivi, visione personale, motivazioni, competenze e sviluppo; gestire i rapporti con gli altri ruoli: operare su interessi e problemi, comunicare la vision, orientarsi al futuro, perseguire successi, costruire coalizioni [8].

L'impostazione del $26^{\circ}$ Congresso Nazionale della SIMeL ha preso spunto da una recente proposta dei ricercatori del Centro di Ricerche sulla Gestione dell'Assistenza Sanitaria e Sociale (CERGAS) Bocconi [9], volta a definire, con il supporto di Siemens Healthcare Diagnostics (Milano, Italia) e del suo Advisory Board, i ruoli dei professionisti, le dinamiche dei servizi e il presidio dei saperi disciplinari di laboratorio, nel quadro delle dinamiche del SSN. Le possibili vocazioni del professionista di laboratorio sono state individuate in: sacerdote della tecnologia (supporto nella selezione di tecnologie e partner, controllo di qualità, valutazione delle forme di gestione); ruolo organizzativo-gestionale (gestione del personale, implementazione di sistemi); consulente clinico (consulenza nella richiesta e nell'interpretazione dei casi singoli); governo clinico [contributo al percorso diagnostico-terapeutico-assistenziale (PDTA), definizione di "pacchetti di prestazione" e progettazione degli screening, formazione di specialisti e medici di medicina generale (MMG)]. L'individuazione dei principali ruoli è stata poi sostenuta dall'indagine condotta presso un gruppo di rappresentanti della professione (n. 249; $57 \%$ al nord; $84 \%$ pubblici; $88 \%$ ospedalieri; $35 \%$ con $<1$ milione esami/anno, $40 \%>1$ e $<3$ milioni esami/anno; $25 \%>3$ milioni esami/anno; $48 \%$ direttori di struttura e $22 \%$ titolari di incarico organizzativo) sulle attività svolte e sulle azioni da intraprendere. Le attività occupano le seguenti percentuali di tempo di lavoro: produzione e validazione dei risultati $30 \%$, organizzazione e gestione $23,2 \%$, scelta delle tecnologie $13 \%$, consulenza su caso clinico $7,4 \%$, ricerca scientifica $5,4 \%$, governo clinico e formazione $10 \%$. Le azioni da intraprendere sono (percentuali di risposta): attività all'interfaccia di gruppo (audit 95\%, rapporti con MMG 94\%, PDTA 94\%) e specifica (referto: format 79\%, commenti $63 \%$ ), gestione (innovazione organizzativa $83 \%$, benchmarking $77 \%$, management $65 \%$ ), formazione e ricerca (corsi $83 \%$, specializzandi $78 \%$, ricerche $75 \%$, epidemiologia 54\%). Secondo questa inchiesta, inoltre, gli elementi che determineranno mutamenti in positivo saranno: crescita culturale dei tecnici sanitari di laboratorio biomedico (TSLB) $89 \%$, progressi dell'automazione $79 \%$, specializzazione delle conoscenze $77 \%$, crescente importanza dell'assistenza territoriale $68 \%$, qualità delle scuole di specialità 55\%; e in negativo: esternalizzazione 
$82 \%$, percezione del laboratorio come commodity $72 \%$, divisione tra le Società scientifiche $71 \%$, scarsità di medici $64 \%$. È interessante notare come i due principali trend della Medicina di Laboratorio nel ventunesimo secolo (centralizzazione e decentralizzazione) abbiano percezioni nettamente e bilanciatamente contrapposte: diffusione dei POCT (point of care testing) 38\% positivo, $32 \%$ negativo, $31 \%$ indifferente; crescita dimensionale dei laboratori $42 \%$ negativo, $40 \%$ positivo, $18 \%$ indifferente. La conclusione della ricerca è l'individuazione di una strategia condivisa nella comunità professionale e centrata sulla necessità del rilancio sulla clinica e sul governo clinico, aprendosi verso l'esterno [10].

La ricerca è di sicuro interesse, individua correttamente i quattro ruoli principali della Medicina di Laboratorio, due interni e due esterni rispetto all'interfaccia clinica-laboratorio, e conferma quanto già noto in merito alle caratteristiche del lavoro del laboratorio, le cause di difficoltà e le possibili azioni [4]. Stupisce il rilievo dato alla cosiddetta "divisione" delle Società scientifiche come fattore negativo di mutamento, anche alla luce delle riflessioni recentemente sviluppate intorno al tema [11], ma evidentemente qui è presente un bias di appartenenza o ideologico. Tuttavia, il quadro dei quattro ruoli è, a nostro avviso, più ricco e complesso: il ruolo di sacerdote della tecnologia comprende innovazione tecnologica, selezione di tecnologie e partner, controllo di qualità, valutazione delle forme di gestione; il ruolo organizzativo-gestionale consiste nella pianificazione strategica, organizzazione e gestione del laboratorio $e$ dei suoi addetti, con particolare attenzione ai processi, al prodotto e al loro controllo; il ruolo di consulente clinico si esplica come consulenza nella richiesta e nell'interpretazione dei casi singoli, ma anche di gruppi interdisciplinari, in particolare a fini di audit clinico; il ruolo del governo clinico si esplica nella definizione collaborativa di "evidenze", linee guida, EBLM (evidence-based laboratory medicine) e PDTA, oltre che nella formazione di specialisti e MMG (Tab. 1).

\section{I contributi del Congresso: ruoli e scenari al tempo} della crisi

Nelle relazioni inaugurali, che hanno dato origine alle Rassegne presentate in questo numero della Rivista, $\mathrm{i}$ quattro ruoli sono stati esaminati sotto i profili dianzi specificati. Mauro Nanni [12], mostrando i cambiamenti intervenuti negli ultimi anni nelle tecniche di una specifica disciplina (la citogenetica), sottolinea le problematiche dell'innovazione, con il suo portato di cambiamento, anche di conoscenze e abitudini operative, e le grandi opportunità di miglioramento non solo dell'efficienza operativa, ma anche dell'efficacia clinica, e il ruolo centrale del professionista nella valutazione, scelta e implementazione della "nuova" diagnostica e nella gestione dei suoi effetti a livello dell'interfaccia clinica-laboratorio. Il ruolo di "sacerdoti della tecnologia" non è puramente relativo alla conoscenza tecnica e alla sua gestione, seppure di qualità, ma ha a che fare direttamente con l'innovazione, nella sua più generale definizione di sviluppo di nuovo valore per gli utenti intermedi e finali, attraverso soluzioni che rispondono a nuovi o non esplicitati bisogni oppure a necessità conosciute, ma con modalità del tutto nuove. "Innovare" in latino significa, infatti, fare qualcosa in modo nuovo, diverso, piuttosto che fare qualcosa meglio. L'innovazione comprende altrettanto bene sia il livello individuale - come creatività, inventiva, capacità di nuove visioni - sia quello organizzativo - come cambiamento delle strutture e obiettivi della produzione e dei suoi processi, progresso delle conoscenze scientifiche e delle competenze correlate e mutamento della percezione, comprensione e atteggiamento psicologico delle persone coinvolte [13]. Margherita Morandini [14], descrive, con esempi tratti dal percorso di rivoluzione organizzativa negli ultimi 15 anni del laboratorio in cui lavora, i contenuti di vision e mission che caratterizzano il ruolo di pianificazione strategica, organizzazione e gestione della struttura, processi e persone del laboratorio. Anche qui l'interpretazione del

Tabella 1 Contenuti dei quattro ruoli principali in Medicina di Laboratorio. In grassetto le principali differenze tra la visione SIMeL e CERGAS

\begin{tabular}{|c|c|c|}
\hline Ruolo del Laboratorio & CERGAS & SIMeL \\
\hline Sacerdote della Tecnologia & $\begin{array}{l}\text { Supporto nella selezione delle tecnologie e dei partner, } \\
\text { controllo qualità, valutazione delle forme di gestione }\end{array}$ & $\begin{array}{l}\text { Innovazione tecnologica, selezione di } \\
\text { tecnologie e partner, controllo di qualità, } \\
\text { valutazione di forme gestionali }\end{array}$ \\
\hline Organizzativo-gestionale & Gestione del personale e implementazione di sistemi & $\begin{array}{l}\text { Pianificazione strategica, } \\
\text { organizzazione e gestione del } \\
\text { laboratorio e del personale }\end{array}$ \\
\hline Consulenza clinica & Consulenza sui casi clinici, audit clinico & Consulenza sui casi clinici, audit clinico \\
\hline Governo clinico & $\begin{array}{l}\text { Contributo a PDTA, definizione "pacchetti prestazioni”, } \\
\text { progettazione degli screening, formazione }\end{array}$ & $\begin{array}{l}\text { Contributo alla costruzione di } \\
\text { "evidenze" (EBLM), linee guida e } \\
\text { PDTA, formazione }\end{array}$ \\
\hline
\end{tabular}

CERGAS, Centro di Ricerche sulla Gestione dell'Assistenza Sanitaria e Sociale; EBLM, evidence-based laboratory medicine; PDTA, percorso diagnostico-terapeutico-assistenziale; SIMeL, Società Italiana Medicina di Laboratorio 
ruolo non è quella banalmente della gestione dell'esistente, ma ha a che fare con la consapevolezza che "strategizing" significa intendere la strategia come pratica, non semplicemente come qualcosa che le organizzazioni hanno (spesso calato dall'alto), ma qualcosa che le organizzazioni fanno nella loro quotidianità. Attualmente è condivisa, infatti, la consapevolezza di dover fondere la "conoscenza" quale principale asset strategico delle organizzazioni con lo strategizing inteso come un processo di apprendimento continuo [15]. Nel quadro attuale per il Laboratorio è necessaria un' "innovazione radicale" e il ruolo del leader, come "trasformatore culturale", è centrale. Annamaria Cenci [16], con ricchissima raccolta di riferimenti bibliografici e operativi, mostra la possibilità di "consulenza" per il laboratorio clinico. Accanto alle forme comunicative tradizionali (il "referto"), per il quale devono peraltro essere definite caratteristiche e capacità di trasmettere adeguatamente e icasticamente le informazioni diagnostiche, si possono ipotizzare due tipologie di consulenza: la consulenza legata al test (counselling), che è un servizio informativo e parzialmente decisionale relativo a un test richiesto da altro specialista, e la consulenza clinica (clinical evaluation), che è una valutazione clinica olistica atta principalmente alla definizione diagnostica del quadro clinico in studio. Per la prima tipologia il laboratorio valuta e interpreta l'esame stesso con gli strumenti di laboratorio, mentre nella seconda il laboratorio è coinvolto nella valutazione e interpretazione dell'appropriatezza, dei percorsi e degli esiti, a volte come protagonista diretto. Il lavoro clinico sul singolo paziente (esame e/o percorso diagnosticoterapeutico) si enfatizza poi in senso generale negli audit clinici. Il ruolo di consultazione della medicina di laboratorio nelle aree cliniche, di ricerca e di salute pubblica è ampiamente riconosciuto nella sanità del mondo occidentale, costituisce un punto essenziale del percorso formativo e rappresenta un momento unitario intra- e interprofessionale [17]. Infine, Romolo Dorizzi et al [18], descrivendo il percorso e gli esiti riorganizzativi della loro realtà (Area Vasta Romagna), dimostrano come la grande massa di dati e di casistica, ottenuta con la concentrazione e la rete $h u b$ \& spokes e gestita accortamente con metodi e sistemi informatici all'avanguardia, consenta di individuare le variabilità di pratica clinica non giustificate dalla popolazione esaminata, di costruire "evidenze" scientifiche e gestionali, di individuare le modalità di intervento e di misurare passo passo l'evolvere delle pratiche e gli esiti sull'efficacia clinica. Misurare in modo accurato, omogeneo e continuo è la base dell'efficienza; misurare numeri significativi di casistica è la base dell'efficacia. Dimensioni e competenze si coniugano per produrre risultati migliori per clinici e pazienti. Da qui nasce una "clinical governance" ade- guata ai tempi della crisi. La storia della modernità e della post-modernità è una storia di velocità, meglio di "accelerazione". Lo stretto rapporto che esiste tra accelerazione e crescita esponenziale determina un accumulo verticale di informazioni, compresse in intervalli di tempo sempre più brevi. Ciò è vero anche per conoscenze e competenze. Basi di dati, efficienza e capacità di visione sono le chiavi per indirizzare il vettore della velocità e controllare l'accelerazione [19].

\section{Conclusioni}

L'insieme delle relazioni mostra la declinazione attuale della Medicina di Laboratorio come intesa dalla SIMeL e schematizzata in Figura 2 e le caratteristiche dei professionisti che si riconoscono nella Società Italiana di Medicina di Laboratorio: apertura all'innovazione e capacità di giudicarla, lavoro all'interno del laboratorio e all'interfaccia del processo di produzione dell'informazione diagnostica, coscienza delle caratteristiche competenziali e innovative dell'equipe del laboratorio, capacità relazionali e collaborative, attori principali della costruzione di "evidenze" scientifiche e gestionali, consapevolezza che i tempi richiedono soluzioni radicali e non piccolo cabotaggio e, soprattutto, il coraggio di veleggiare nei mari dell'incertezza ma delle opportunità. Dice Peter Drucker: "La differenza tra anticipare il futuro e subirlo sta tutta nel coraggio con cui lo si affronta per tempo" [20].

Conflitto di interesse Nessuno

\section{Bibliografia}

1. Cappelletti P (2004) Un Patto per la Modernizzazione e la Umanizzazione della Medicina di Laboratorio in Italia. Riv Med Lab - JLM 5:252-254

2. Cappelletti P (2012) Medicina di Laboratorio e Postmodernità. Riv Ital Med Lab 8:1-15

3. Cappelletti P (2012) Il futuro della Sanità: sostenibilità finanziaria e scenari evolutivi. Riv Ital Med Lab 8:63-70

4. Cappelletti P (2012) Medicina di Laboratorio: un futuro possibile. Riv Ital Med Lab 8:121-130

5. Christensen CM, Overdorf M (2000) Meeting the challenge of disruptive change. Harv Bus Rev 2:66-76

6. Cappelletti p (2010) Medicina di Laboratorio. In: Galzigna L, Plebani M (Eds). Trattato Italiano di Medicina di Laboratorio. Volume 1: Biochimica Clinica Generale. Piccin Nuova Libraria, Padova

7. Plebani M, Laposata M, Lundberg GD (2011) The Brain-to-Brain Loop concept for laboratory testing 40 years after its introduction. Am J Clin Pathol 136:829-833

8. Rotondi P, Saggin A (2002) Persona e organizzazione. Sviluppare competenze per valorizzarsi in sanità. McGraw-Hill, Milano

9. Longo F (2011) Scenari per la sanità futura: quale strategia di ruolo per i dirigenti di laboratorio? In: Scenari sulla trasformazio- 
ne del capitale umano. Workshop Siemens Healthcare Diagnostics. $1^{\circ}$ Congresso Nazionale della Medicina di Laboratorio, Parma 15-18 novembre 2011. http://www.congressomedicinalaboratorio.it/images/stories/pdf/A11_007_ProgrammaDefinitivo.p df [Accesso 10 luglio 2012]

10. Salvatore D (2012) Maturare le riflessioni nella comunità ed implementare azioni. Workshop Siemens Healthcare Diagnostics. $26^{\circ}$ Congresso Nazionale SIMeL, Salerno 3-5 ottobre 2012. http://www.simel.it/it/convegno.php/104549 [Accesso 10 novembre 2012]

11. Cappelletti P (2011) Il future delle Società Scientifiche e la Società Italiana di Medicina di Laboratorio. Riv Ital Med Lab 7:127-139

12. Nanni M (2012) Scenari e ruoli della Medicina di Laboratorio al tempo della crisi: i sacerdoti della tecnologia. Riv Ital Med Lab DOI: $10.1007 / \mathrm{s} 13631-012-0076-6$

13. Von Hippel E (1988) The Sources of Innovation. Oxford University Press, Oxford
14. Morandini M (2012) Scenari e ruoli della Medicina di Laboratorio al tempo della crisi: pianificazione, organizzazione e gestione. Riv Ital Med Lab DOI: 10.1007/s13631-012-0075-7

15. Jarzabkowski P, Spee AP (2009) Strategy-as-practice: a review and future directions for the field. Int J Manag Rev 11:69-95

16. Cenci AM (2012) Scenari e ruoli della Medicina di Laboratorio al tempo della crisi: consulenza clinica. Riv Ital Med Lab DOI: 10.1007/s13631-012-0073-9

17. Marques MB, Anastasi J, Ashwood E et al (2011) The Clinical Pathologists as Consultant. Am J Clin Pathol 135:11-12

18. Dorizzi RM, Baldrati L, Vascotto L et al (2012) Scenari e ruoli della Medicina di Laboratorio al tempo della crisi: governance clinica, EBLM, line guida e PDT/PA. Riv Ital Med Lab DOI: 10.1007/s13631-012-0074-8

19. Eriksen TH (2001) Tyranny of the moment. Fast and slow time in the information age. H Aschehoug \& Co, Oslo

20. Drucker PF (2007) The essential Drucker. Taylor \& Francis Group, Philadelphia 